\title{
Gute Zertifizierungspraxis - eine Initiative der DGHO
}

\author{
Mathias Freund \\ Klinik für Innere Medizin III, Universitätsmedizin Rostock, Deutschland
}

Immer mehr Kliniken und Praxen streben den Erwerb eines Zertifikates an, um damit ihre Kompetenz in der Onkologie nachzuweisen und ihrer Einrichtung ein besonderes Profil zu geben. Die Zertifizierung bestätigt die Konformität eines Unternehmens (bzw. eines Produktes, einer Dienstleistung und anderen) mit einer normativen Anforderung. Dies erfordert die externe Überprüfung einer definierten Struktur-, Prozess- und Ergebnisqualität im Rahmen eines Zertifizierungsverfahrens. Dabei werden schwerpunktmäßig die Strukturen und Vorgehensweisen innerhalb eines Hauses unter die Lupe genommen: die Organisationsbereiche, das Qualitätsmanagement, die räumliche Ausstattung, die Dokumentation, die Leitlinien-Adhärenz und vieles mehr. Die Ergebnisqualität ist ungleich schwerer zu überprüfen und zu beurteilen, denn harte Erfolgsparameter - etwa eine erhöhte Heilungsrate oder eine bessere Lebensqualität der Patienten - sind in Ermangelung flächendeckender Krebsregister kaum zu erheben. Behelfsweise können Surrogatparameter - zum Beispiel die Komplikationsrate nach onkologischen operativen Eingriffen, die Infektionsrate oder die Häufigkeit von Rehospitalisierungen - hinzugezogen werden. Diese Daten können aber in Abhängigkeit von den Patientencharakteristika und der speziellen Ausrichtung der onkologischen Einrichtungen sehr unterschiedlich ausfallen. Folglich lässt sich die Frage, ob eine erfolgreiche Zertifizierung mit einem besseren Behandlungsergebnis korreliert, anhand der verfügbaren Daten nicht pauschal beantworten. Hilfreich sind womöglich retrospektive Analysen auf Basis einer ausreichend großen Zahl von Patienten, die aber erst in einigen Jahren zur Verfügung stehen dürften.

Auch wenn noch offen ist, woran die Qualität eines Onkologischen Zentrums am besten festgemacht werden kann, erleben wir zurzeit einen regelrechten Zertifizierungsboom. Ein Zertifikat ist ein begehrtes Qualitätssiegel im Wettbewerb der Einrichtungen. Zeichnet sich das Zertifikat durch Alleinstellungsmerkmale aus - was durch stete Heraufsetzung der Anforderungen zu erreichen ist -, scheint dies den Wert noch zu steigern. Daraus hat sich ein regelrechter Markt für
Zertifizierungsberater und Zertifizierungsunternehmen entwickelt sowie ein Rennen medizinischer Fachgesellschaften um immer neue und anspruchsvollere Zertifikate. Dies ist eine durchaus bedenkliche Entwicklung, die man aber zur Kenntnis nehmen muss.

Die Zertifizierung birgt somit potenzielle Interessenkonflikte der Akteure: Es gibt finanzielle Interessen der Zertifizierungs- und Beratungsunternehmen, das Profilierungsinteresse der Einrichtungen, aber auch berufspolitische Interessen der Fachgesellschaften. Das unterstreicht die Notwendigkeit, den Zertifizierungsprozess einer Qualitätssicherung zu unterziehen und die Anbieter zur Einhaltung einer guten Zertifizierungspraxis zu verpflichten. Die Deutsche Gesellschaft für Hämatologie und Onkologie (DGHO) hat vor diesem Hintergrund die Charta «Gute Zertifizierungspraxis» ausgearbeitet, die sich als Ergänzung zu weiteren Qualitätssicherungsmaßnahmen (namentlich die Akkreditierung der Zertifizierer durch die Deutsche Akkreditierungsstelle (DAkkS)) versteht.

Unser Anliegen war es dabei, die allgemeinen Standards der Qualitätssicherung von Zertifizierungsverfahren auf die Besonderheiten medizinischer Zertifizierungsverfahren $\mathrm{zu}$ übertragen. Grundlagen der Zertifizierung sind Objektivität und Transparenz, Sachorientierung und Unabhängigkeit der Überprüfung (Abb. 1).

Sowohl an das Zertifizierungsunternehmen als auch an die Fachexperten, die im Rahmen einer Zertifizierung hinzugezogen werden, sind grundlegende Anforderungen zu stellen.

Für das Zertifizierungsunternehmen gilt:

- Der Zertifizierer ist «Herr des Verfahrens» und entscheidet über die Zertifizierung.

- Der Zertifizierer muss über eine dem Zertifizierungsbereich entsprechende Akkreditierung bei der DAkkS verfügen.

- Der Zertifizierer soll aus Gründen des Wettbewerbs keine exklusiven Verträge mit Fachgesellschaften abschließen.

- Die Auswahl des Zertifizierungsunternehmens liegt beim Kunden (Klinik, Praxis).

\section{KARGER}

Fax +49 7614520714

Information@Karger.de

www.karger.com 


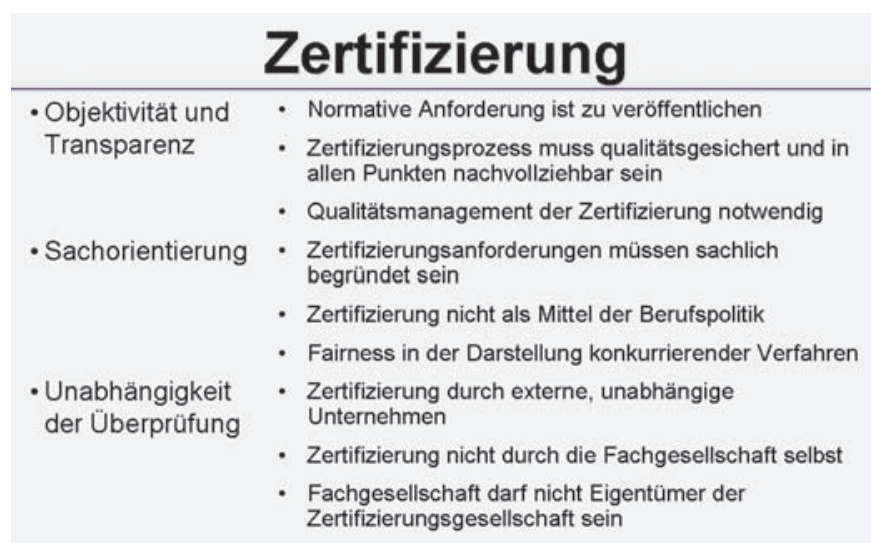

Abb. 1. Prinzipien der Zertifizierung.

- Der Zertifizierer führt keine Beratung im Vorfeld einer Zertifizierung durch.

- In medizinisch-fachlichen Fragen kann bei Bedarf eine Ratifizierung durch eine Zertifizierungskommission (bzw. Zertifikaterteilungskommission) erfolgen.

Für Fachexperten gilt:

- Die Ernennung von Fachexperten erfolgt qualitätsgesichert nach definierten Kriterien.

- Ihre fachliche Eignung ist durch Fachgesellschaften zu bestätigen.

- Die Fachexperten werden regelmäßig geschult.

- Eine Liste der Fachexperten steht allen Zertifizierungsunternehmen zur Verfügung.

- Exklusivverträge zwischen Experten und Zertifizierungsunternehmen sind unzulässig.

- Die Auswahl der Fachexperten für ein konkretes Zertifizierungsverfahren erfolgt durch das Zertifizierungsunternehmen.

Künftig dürfte die Wirtschaftlichkeit und Transparenz von Zertifizierungen stärker in den Vordergrund treten, zumal mittlerweile eine Vielzahl von Zertifizierungsunternehmen miteinander im Wettbewerb steht. Auch zu diesen Aspekten können einige grundlegende Prinzipien formuliert werden (Abb. 2).

\section{DGHO-Initiative «Gute Zertifizierungspraxis»}

Die DGHO setzt sich für die Einhaltung der beschriebenen Standards für die Zertifizierung ein. Sie hat im November 2010 die Initiative «Gute Zertifizierungspraxis» gestartet und diese wenige Monate später bei der Arbeitsgemeinschaft der Wissenschaftlichen Medizinischen Fachgesellschaften e.V. (AWMF) vorgestellt. Daraufhin führte ein AWMF-Beschluss im November 2011 zur Bildung der Arbeitsgruppe «Gute Zertifizierungspraxis», die sich mit der weiteren Ausformulierung des Charta-Entwurfes zur Einhaltung bestimmter Vorgehensweisen im Zuge von Zertifizierungen im Gesundheitswesen (s. Anhang, auch einsehbar unter www.dgho.de) befassen wird.

\section{Wirtschaftlichkeit und Transparenz}

- Unwirtschaftlichen und ineffizienten Zertifizierungsverfahren entgegentreten

- Vergabe von Beratungsleistungen zur Unternehmensstrategie von Beratungsleistungen zur Zertifizierung trennen

- keine Provisionszahlungen von Zertifizierungsunternehmen an die Beratungsunternehmen

- keine Zahlungen der Zertifizierungsunternehmen an die Fachgesellschaft

- Transparente Preisgestaltung der Zertifizierungsunternehmen (z.B. veröffentlichte Preislisten)

Abb. 2. Grundbedingungen für Zertifizierungsunternehmen.

\section{Wirtschaftlichkeit und Transparenz durch offene und neutrale Zertifizierungsplattform} www.zertifzierung-onkologie.de

- Grundsatzpapiere und Anforderungen

- Internet-basierte Abwicklung der Zertifizierungsprojekte

- Bereitstellung der QM-Systeme für die Zertifizierung

- Darstellung der zertifizierten Zentren

- Leistungszahlen zertifizierter Zentren (Benchmarking)

Abb. 3. Zertifizierungsplattform: neutrale Informationen, offener Austausch.

\section{Informationsportal für Zertifizierungen}

Ein weiteres, aus der Initiative zur Guten Zertifizierungspraxis entstandenes Projekt nutzt das Internet als Kommunikationsplattform zu Fragen bezüglich Zertifizierungsverfahren. Wir wollen mit der Etablierung dieses übergreifenden Informationsangebotes unter www.zertifizierung-onkologie.de sowie www.zertifizierung-medizin.de einen offenen Austausch der Fachgesellschaften und Zertifizierungsunternehmen zu Fragen der Zertifizierung ermöglichen (Abb. 3).

Insbesondere die internetbasierte Abwicklung von Zertifizierungsvorhaben dürfte den Prozess wesentlich vereinfachen und beschleunigen. Die am jeweiligen Zertifizierungsverfahren Beteiligten können über eine Datenbank ihre Arbeitsergebnisse eingeben und bearbeiten (zum Beispiel in Vorbereitung eines Audits). Auch Arbeitslisten für Begehungen, Stellungnahmen und Gutachten lassen sich auf diese Weise speichern und von allen Projektteilnehmern rasch abrufen. Ein weiterer Bereich umfasst die Darstellung der relevanten Vorgehensweisen und Qualitätsmanagement(QM)-Systeme für die Zertifizierungsverfahren sowie eine Listung bereits zertifi- 
zierter Zentren. Andere medizinische Fachgesellschaften können bei Bedarf die Struktur dieser Plattform für die Darstellung ihrer eigenen Zertifizierungsangebote nutzen. Die DGHO möchte damit auf andere Anbieter von Zertifizierungsverfahren zugehen und einen Beitrag zur Sachorientierung im bis dato recht undurchschaubaren Bereich der Zertifizierung leisten.

\section{Disclosure Statement}

Es bestehen keine Interessenkonflikte.

\section{Anhang: Charta "Gute Zertifizierungspraxis", Entwurf der DGHO vom 03.11.2010}

Gute Praxis bei der Durchführung von Zertifizierungen in der Medizin

\section{Präambel}

Zertifizierungen und die damit verbundene Überprüfung von Struktur- und Ergebnisqualität sind zweifellos positive Errungenschaften im Gesundheitswesen. Durch sie sollen dem Patienten eine höhere Qualität medizinischer Leistungen und bessere Ergebnisse zugute kommen.

Sie binden aber auch personelle und materielle Ressourcen, die bei begrenztem Budget mit den Mitteln für die unmittelbare Betreuung der Patienten konkurrieren. Die Fachgesellschaften sind daher aufgefordert, in hohem Maße Verantwortung für die Qualität, die Effizienz und Wirtschaftlichkeit von Zertifizierungen zu übernehmen.

In diesem Sinne verpflichten sich die Unterzeichner dieser Charta zu einer guten Praxis bei der Durchführung von Zertifizierungen in der Medizin.

\section{Grundlagen der Zertifizierung}

Eine Zertifizierung bestätigt die Konformität eines Unternehmens, eines Produktes, einer Dienstleistung usw. mit einer normativen Anforderung.

- Diese normative Anforderung ist eindeutig und öffentlich zugänglich im Sinne eines Grundsatzpapiers zu definieren. Zertifizierung bedeutet Überprüfung durch eine unabhängige externe Institution.

- Die Zertifizierung kann nicht durch Fach- oder Dachgesellschaften selbst erfolgen. Interessenkonflikte wären in diesem Falle wegen eventueller beruflicher Interessen ihrer Mitglieder nicht auszuschließen.

- Zertifizierung darf nicht als Mittel der Berufspolitik verwendet werden.

\section{Stellung des Zertifizierungsunternehmens}

- Das Zertifizierungsunternehmen ist Herr der Zertifizierung und entscheidet über die Erteilung oder Verweige- rung des Zertifikats. Nur so ist die Unabhängigkeit des Verfahrens gewährleistet.

- In der Medizin werden fachliche Aspekte wesentlich berührt. Daher ist eine Ratifizierung der Entscheidung des Zertifizierungsunternehmens durch ein fachliches Gremium wie zum Beispiel eine Zertifizierungskommission sinnvoll. Bei der Errichtung eines solchen fachlichen Gremiums ist auf Transparenz und Ausgewogenheit zu achten.

- Das Zertifizierungsunternehmen darf keine Beratungsleistungen in Institutionen erbringen, die später durch das Unternehmen zertifiziert werden sollen.

\section{Auswahl des Zertifizierungsunternehmens}

- Die Auswahl des Zertifizierungsunternehmens obliegt demjenigen, der den Antrag auf Zertifizierung stellt. Eine exklusive Bindung eines Zertifizierungsverfahrens an einen oder mehrere Zertifizierer ist nicht zulässig.

- Für die Zertifizierung sollen nur Zertifizierungsunternehmen herangezogen werden, die ihre Qualität durch Akkreditierung bei der auf gesetzlicher Grundlage errichteten Deutschen Akkreditierungsstelle (DAkkS) nachweisen können. Der Zertifizierungsbereich soll mindestens umfassen: M38 = Gesundheits-, Veterinär- und Sozialwesen, T00 $=$ Qualitätsmanagementsystem (QMS/ISO 9001).

\section{Fachexperten}

Die Zertifizierungsgesellschaften benötigen für die Durchführung der Zertifizierung Experten mit den entsprechenden Fachkenntnissen.

- Die Ernennung der Fachexperten muss qualitätsgesichert erfolgen. Die Fachexperten sollen eine entsprechende Anerkennung durch die Fachgesellschaften haben. Sie sind regelmäßig zu schulen.

- Die Liste der anerkannten Fachexperten ist den im Gebiet aktiven Zertifizierungsunternehmen zugänglich zu machen.

- Die Zertifizierungsgesellschaften verpflichten die Fachexperten zur Durchführung der Zertifizierung.

\section{Wirtschaftlichkeit und Transparenz}

Die Fachgesellschaft

- verpflichtet sich, unwirtschaftlichen und ineffizienten Zertifizierungsverfahren entgegenzutreten. Zertifizierungen sollen in möglichst großen Einheiten zeitlich abgestimmt und nicht kleinteilig erfolgen, um den Ressourcenverbrauch zu begrenzen.

- empfiehlt Institutionen, die Vergabe einer Beratungsleistung zur Unternehmensstrategie und Vorbereitung eines Zertifizierungsverfahrens strikt von der Vergabe von Leistungen bei der Errichtung von Qualitätssicherungssystemen und Ähnlichem zu trennen. So wird der Gefahr entgegengetreten, dass eine Empfehlung zu kleinteiligen oder verzettelten Zertifizierungsverfahren durch hohe nachfolgende Auftragsvolumen an den gleichen Berater stimuliert wird. 
- verpflichtet die Zertifizierungsunternehmen, keine Provisionen an Beratungsunternehmen zu zahlen, die im Vorfeld von Zertifizierungen tätig waren.

- verpflichtet sich selbst, keine Zahlungen von Zertifizierungsunternehmen anzunehmen.

- darf nicht Eigentümer eines Zertifizierungsunternehmens sein, das in ihrem Einflussbereich aktiv ist.

- verpflichtet die in ihrem Bereich tätigen Zertifizierungsunternehmen zu Transparenz ihrer Preisgestaltung durch öffentliche Preislisten.

\section{Objektivität und Fairness in der Darstellung von}

Zertifizierungsverfahren

- Die unterzeichnenden Fachgesellschaften sind einem übergreifenden Anspruch auf Objektivität verpflichtet und fördern den Gedanken der Zertifizierung und Qualitätssicherung. Sie verpflichten sich daher, über konkurrierende Modelle in der Zertifizierung objektiv und sachlich zu berichten und zu informieren und andere Fachgesellschaften im Sinne dieser Charta zu unterstützen. 